\title{
EL COMERCIO ELECTRÓNICO COMO HERRAMIENTA DE COMERCIALIZACIÓN PARA LOS EMPRESARIOS ${ }^{1}$
}

E-COMMERCE AS A TOOL FOR MARKETING FOR EMPLOYERS

\author{
Rosa María González López ${ }^{2}$ \\ Lilia Vázquez Martínez ${ }^{3}$ \\ Jorge Pérez Mejía ${ }^{4}$
}

Para citar este artículo: González, R., Vásquez, L. y Pérez, J. (2014). "El comercio electrónico como herramienta de comercialización para los empresarios". Revista Inquietud Empresarial. Vol. XIV (1), p.p. 115-128

Fecha de recepción: 02 de febrero de 2014 Fecha de Aceptación: 20 de mayo de 2014

1 Artículo de Reflexión como resultado del proyecto de investigación "El comercio electrónico como herramienta de comercialización para los empresarios" realizado por el grupo de investigación Gestión de la Política de Educación Superior en México e Innovación y Desarrollo Organizacional.

2 Doctora en Derecho Laboral de la Benemérita Universidad Autónoma de Puebla. México Docente investigadora de la Facultad de Administración de la Benemérita Universidad Autónoma de Puebla. rossyadmon@gmail.com

3 Maestra en Administración de Pequeñas y Medianas Empresas de la Benemérita Universidad Autónoma de Puebla. México. Docente investigadora de la Facultad de Administración de la Benemérita Universidad Autónoma de Puebla. liliavazm@yahoo.com.mx 4 Doctor en Educación de la Benemérita Universidad Autónoma de Puebla. México. Docente investigador de la Facultad de Administración de la Benemérita Universidad Autónoma de Puebla. jpmejiabuap@gmail.com 


\section{RESUMEN}

El objetivo de este artículo es sensibilizar a los empresarios, en el uso del comercio electrónico para ofrecer sus servicios y productos a través del internet, pues el mercado no es lo suficientemente grande como para impulsar un crecimiento en sus índices de facturación, situación que puede verse favorecida con el nuevo auge de las firmas virtuales. El Comercio Electrónico (E-Commerce), concebido en un principio como medio que complementa otras formas de comercio, ahora se proyecta como una tecnología para el cambio, que por su relación costo-beneficio está al acceso de cualquier organización.

Cada día se fortalece la cultura para comprar por medio electrónico y será más favorable su aceptación, ya que las generaciones jóvenes están creciendo dentro de un esquema diferente de comercializar y estarán más habituados al Comercio Electrónico. Para ello, se debe poner más atención a la vigencia y funcionamiento del Código de Comercio en México, el cual se encarga de regular los negocios electrónicos en el país.

\section{PALABRAS CLAVE}

E-Commerce, Tecnología, Páginas Web, Tendencia, Cultura

\section{ABSTRACT}

The purpose of this article is to raise awareness among employers on the use of e- business to offer their services and products through the internet, because the market is not big enough to promote growth in their turnover rates, a situation that can be favored with the new growth of virtual signatures. Electronic Commerce (E -Commerce ), initially conceived as a supplementary mean of other forms of commerce, is now being projected as a technology to make a change, that by their cost- benefit is available to all companies.

Each day the culture to buy electronic means gets strengthen which results in a more favorable acceptance, this is because the younger generations are growing up in a different scheme to trade and will be more accustomed to electronic commerce. In order to achieve this more attention must be paid to the life and operation of the Code of Commerce in Mexico, which is responsible for regulating electronic business in the country

\section{KEYWORDS}

E-Commerce, Technology, Web Pages, Trend, Culture 


\section{INTRODUCCIÓN}

Según López y Pérez (s.f), en la actualidad el comercio electrónico es una de las formas de hacer negocios, por esta razón muchas empresas que nacieron en la economía tradicional han tenido que preocuparse por modificar su estrategia de negocios y así alinearse a las oportunidades que ofrece el comercio electrónico, pues en este medio, la velocidad es tan importante en el proceso de creación como durante la gestión diaria de las empresas.

Una vez que una organización sale al mercado debe operar a la velocidad de la red. Con el crecimiento de las empresas basadas en el comercio electrónico, la gente ve la oportunidad de generar sus propias políticas en mercados que en un principio pasaron de ser locales, a regionales, y finalmente a mundiales.

"Por parte de la empresa, el panorama es mucho más alentador que por parte del cliente, pues las empresas consideran que el E-Commerce les permitiría reducir los inventarios, hacer más fáciles las relaciones con los clientes, llegar a un mercado más amplio, etc" (Moreno, s.f).

Aunque el Comercio Electrónico no crecerá al mismo ritmo de otras naciones como es el caso de los países europeos o como el país vecino del Norte, eso no indica que vaya a decrecer en el corto, mediano o largo plazo; pues se deberá ser cuidadoso en los puntos básicos de información que corresponderá tener el sitio web, que pretenda dedicar sus actividades al comercio electrónico.

\section{Referentes teóricos}

En México se han realizado diferentes avances por parte de particulares de alcanzar un esquema estructurado para el ejercicio del comercio electrónico, por ejemplo en 1986 se conformó la Asociación Mexicana de Estándares para el Comercio Electrónico (AMECE, la cual promueve el uso de normas y los posibles beneficios para el desarrollo de dicho comercio (Lazcano, 2006) . Ésta se encuentra respaldada por organizaciones internacionales como Uniform Code Council (UCC) de los Estados Unidos de Norteamérica y, International Article Numering Association ( EAN).

Dicha asociación civil, promueve principalmente estándares como Código de Barras o Código de Producto, Número de Localización EAN e Intercambio Electrónico de Datos, denominado EDI. "Esto gracias al trabajo que conjuntamente desarrolla mediante comisiones específicas como: el Comité de Identificación, integrado por grupos de trabajo Comercial y el Catálogo Electrónico de Productos, encabezado por Ramón Vélez de Industrias Vinícolas Pedro Domecq. El Comité EDI México tiene como objetivo establecer la administración de los estándares para el Intercambio Electrónico de Datos, sobre la base de las necesidades de distintos sectores comerciales, industriales y de servicios" (Lagarde, s.f).

Un estudio elaborado a finales del 2000 por Cisco Systems y la Universidad de Texas expresa que la economía de Internet sólo en Estados Unidos es de tal magnitud, que podría calificarse como una de las mayores del mundo, situada después de Suiza en términos de producto interno bruto (Carrol y Broadhead, 2000). 
Los índices de facturación de organizaciones como eBay y Amazon han llevado a los analistas a pensar que se aproxima una nueva era de las organizaciones virtuales. Por otro lado, como explica Benchmark, la cantidad de empresas enfocadas en ofrecer servicios y productos a través de la red se ha reducido considerablemente, y el mercado no es lo suficientemente grande como para impulsar un crecimiento similar al que se registró entre 1998 y 1999.

De cualquier manera, los especialistas aseguran que la caída del sector en esos años trajo consigo una depuración de las compañías especializadas en la virtualidad, lo que augura un panorama mejor definido y más estable para las 'Puntocom'.

Desde siempre, los autores y expertos han buscado conceptos y definiciones del Comercio Electrónico, coincidiendo que este comenzó antes de Internet, mediante transacciones comerciales por télex, teléfono y fax, pero el desarrollo de la WEB global motivó que alcanzara mayor auge, por su masividad y rapidez de operación (Redherring Communications, 2001).

Para especialistas integrantes de la Comisión Nacional de Comercio Electrónico de Cuba, puede definirse como "cualquier forma de transacción de negocios en la cual las partes interactúan electrónicamente mediante intercambios materiales o contacto físico directo", y agrega que su esencia se capta mejor si afirmamos que es "uno de los casos poco frecuentes en que se unen las recientes necesidades con las tecnologías nuevas para revolucionar la forma en que se realizan los negocios" (Fernández, 2000).

Desde esta óptica se pueden establecer dos categorías dentro del Comercio Electrónico: Indirecto y Directo. En el primero, se realiza la selección y pedido de diferentes mercancías por medios electrónicos y se hacen llegar al cliente final por los canales tradicionales, lo cual requiere logística de apoyo junto con transportes e infraestructura.

En la segunda, categoría, el pago se realiza totalmente en línea, a través de la misma red global, lo cual disminuye los costos para el fabricante o distribuidor. De esta manera, este tipo del llamado Comercio Electrónico sólo se lleva a cabo, con mercancías llamadas "intangibles", cuyo ciclo de venta, pago y entrega final puede adelantarse por Internet como Videos, fotos, libros, revistas, programas de computación y música.

\section{Generalidades del Comercio Electrónico}

El comercio electrónico lo entendemos tanto en la compra de productos o servicios en Internet, como la transferencia electrónica de datos entre operadores de un sector en un mercado o en intercambio de cantidades o activos entre entidades financieras, o la consulta de información, con fines comerciales, a un determinado servicio, o un sinfín de actividades de similares características realizadas por medios electrónicos (Cámaras, 2005).

El comercio electrónico es una de las formas del proceso de integración de las Tecnologías de la Información en las organizaciones. Sin duda, una de las más amplias y de fácil acceso y, por tanto, la de mayor visibilidad. La integración total da lugar al e-business, estadio en el que las TIC son utilizadas en todas las actividades de una empresa, no sólo en las de venta (e-commerce). 
En la actualidad, casi cualquier persona sin importar su edad y condición social, sería capaz de citar al menos una web, dónde poder comprar bienes o servicios. El comercio electrónico ha evolucionado convirtiéndose en una forma de comprar, y cada vez se usa de manera más habitual tanto en la computadora (entendido este como dispositivo clásico de acceso a la WWW) como en dispositivos móviles. Sin embargo, la compra en línea todavía se percibe como algo novedoso y, a menudo, inseguro. Es habitual usar el internet como sistema informativo, pero que la adquisición (sobre todo bienes) se hace en el establecimiento físico. Así pues, es necesario un gran esfuerzo que deben hacer los sistemas de compra online (sistemas confiables y muy seguros).

A priori, las principales ventajas del comercio electrónico frente a sistemas de compra tradicional para el cliente serían: precios más bajos (costos de logística), stock superior, el lugar para la búsqueda de información relacionada con los productos muy amplia. De cara al proveedor del bien o servicio, la principal ventaja sería "el acceso universal" al mercado a niveles similares para cualquier empresa y con costes de implantación menores que la implantación física.

$\mathrm{El}$ hecho de que contemos con varios niveles de confianza -las redes sociales pueden ir de amigos a gente desconocida- afecta a nuestras decisiones de compra y, por tanto, un buen sitio de comercio electrónico debe ser capaz de incidir en las redes de usuarios activos que participan, opinan y aportan diálogo con el objetivo de incidir en nuestras decisiones.

El comercio electrónico es ya de facto un sistema de compra que convive con los anteriores, pero que irá ganando adeptos -y será mayor entre la gente de menor edad en el futuro-. A medida que el comercio electrónico se extiende a dispositivos móviles y aparecen tarjetas exclusivas/optimizadas para uso electrónico, su peso irá aumentando frente a otros sistemas de pago (Kenneth, y Carol, 2012).

México posee un número elevado de usuarios de Internet, en total más de 40 millones de personas ya se conectan a la Red de Redes y en cuanto a las entidades federativas con la mayor cantidad de usuarios son el Distrito Federal, estado de México y el estado de Jalisco. El tiempo diario promedio de navegación de los usuarios es de 4 horas con 9 minutos (casi media jornada laboral) y los días en los que hay mayor uso de la red son los lunes y viernes y el $84 \%$ de las empresas que utilizan Internet tienen contratado a un Community Manager mientras que 8 de cada 10 empresas tienen un perfil activo en Redes Sociales $(92 \%$ en Facebook y $86 \%$ en Twitter) (ILifebelt Times, 2013).

Según Poder Edomex (2013), el 92\% de los ciudadanos mexicanos quienes tienen acceso a Internet acceden de forma regular a una Red Social, el restante $8 \%$ no visita estos espacios por falta de Interés, por Protección de Datos y por Falta de Tiempo, $64 \%$ de estos usuarios son mayores de edad. El mercado de las Redes Sociales está distribuido de la siguiente forma: 90\% de quienes usan internet tiene cuenta en Facebook (de hecho las estadísticas de Facebook afirman que actualmente hay más de 41 millones de mexicanos con un perfil activo en esta red social), $60 \%$ tiene cuenta en Youtube, 56\% tiene cuenta en Twitter, 34\% en Google+ y 25\% en Hi5.

En 2002, había 78 millones de empresas con páginas WEB y las inversiones de compañías y corporaciones en iniciativas de Internet sólo EE.UU, que en 1999 totalizaron 85 mil millones de dólares, se incrementaron un 139\% (Carrol y Broadhead, 2000). 
Las ventas del comercio electrónico en México cerraron en 2012 con un crecimiento de $46 \%$ respecto al 2011 y representarán transacciones por más de 6,000 millones de dólares, proyecta la Asociación Mexicana de Internet (Amipci) en su Estudio de Comercio Electrónico en 2013.

Según Mauricio Braverman, vicepresidente ejecutivo de la Amipci, existen en México más de 14 millones de compradores habituales en línea, que han ido desarrollando la confianza de adquirir bienes y servicios en línea y con acceso a una oferta más diferenciada de productos respecto de años anteriores.

"Del 2010 al 2012, la industria del comercio electrónico se duplicó. Pocas industrias pueden hablar de estos saltos", detalló Braverman al presentar el estudio. "Han crecido los métodos de pago, los internautas, cada vez los jóvenes compran más, la confianza está en aumento y la oferta de productos ha ido en crecimiento".

Braverman sostuvo que aún existe una oportunidad de desarrollo mucho mayor en el sector, puesto que aún no se llega a los niveles de crecimiento de algunos países latinoamericanos como Brasil, cuya industria de comercio digital, en palabras del ejecutivo, es cinco veces mayor a la mexicana.

\section{Incremento de los Compradores en línea}

"El Estudio de Comercio Electrónico 2012 de la Amipci destaca que alrededor de 35\% de los internautas compran bienes o contratan servicios cada mes, lo que representa un crecimiento de $5 \%$ respecto al año previo. $34 \%$ de quienes adquiere productos o servicios lo hace de forma trimestral, explicó Renato Juárez, vicepresidente de Investigación de la Amipci” (El Economista, 2012).

Otro aspecto sobresaliente es que $47 \%$ de los internautas evaluados ha realizado compras por medio de un dispositivo móvil, lo que significa un avance significativo de 21 puntos porcentuales respecto a este mismo indicador en el estudio del 2011.

La Asociación Mexicana de Internet (AMIPCI) en 2012 publicó la siguiente gráfica:

Figura 1. Crecimiento del comercio electrónico en México



Fuente: Asociación Mexicana de Internet (AMIPCI) (2012). 
En rubros como reservaciones turísticas y de pasajes, fotos, videos, música, información y software, no se requiere infraestructura logística, porque la distribución y pago se realiza directamente por Internet. Entre empresas, su uso ya es muy importante, y sectores como banca, medicina, seguros, etc., ya hacen usos intensivos de servicios online con incluso más prestaciones que los equivalentes presenciales. Algunas plataformas de pago (como PayPal) ya nos permiten operar de forma global con cualquier sistema de pago aceptado y serán tan indispensables en el futuro como ahora son los bancos.

Las garantías de un sistema de ventas online, es fundamental para valorar los aspectos clave del negocio en términos de su paso a internet, con interrogantes como: si el stock estará al día, el catálogo de productos se encuentra bien definido, si contaremos con un sistema para la entrega de productos fiable, qué sistemas de pago se aceptarán, cómo se va a promocionar el comercio en la red o si tendremos un boletín informativo diario, semanal o mensual con ofertas. Éstas son cuestiones que no deberían dejar lugar a dudas (Liberos y García, 2012).

En algunos casos, el paso al e-commerce puede ser más complejo que en otros, pero lo fundamental es tener un plan de negocio que ayude a su implementación. En dar el paso al e-commerce, parece ser que el último gran interrogante por resolver es el producto virtual o intangible. Concretamente, el producto "información" es el que mayores debates sigue suscitando sobre los modelos de negocio en internet y su viabilidad.

Las dificultades jurídicas son consecuencia inmediata del dominio del papel escrito en el derecho de todos los estados de lo que podíamos llamar nuestro ámbito de responsabilidad, y de las obligaciones impuestas, por el derecho de esos estados, de expedir y de conservar los documentos, en términos generales sobre soporte papel, con una fuerza jurídica especial que recibe en la característica manuscrita de la firma que los ordenamientos jurídicos a los que nos referimos considera necesaria para satisfacer las condiciones de validez o de negociabilidad de un documento (Cámaras, 2005).

Actualmente, en todo comercio electrónico debe existir un canal de distribución electrónico que, en algunos casos, deberá ser completado con un canal de distribución tradicional que permita llegar los bienes y productos que se han contratado desde el productor o desde el distribuidor, hasta el usuario; el canal de distribución que en sí puede ser la red solamente se configura como un canal de distribución de datos o de información en los que se puede o no incluir un compromiso electrónico que deberá reunir unas determinadas características.

Existen dos aspectos a considerar en el Comercio Electrónico: el error y el fraude. La diferencia entre uno y otro seria la intencionalidad pero el resultado podría ser el mismo desde la óptica de la autenticidad del contenido y de la identidad del usuario, surgiendo rápidamente: los documentos que han sido recibidos por medios telemáticos y posiblemente procesados por un ordenador, ¿en que medida son fiel reflejo de la información que originalmente contenían? (Financial Times online, 2001). 


\section{Características del Pago Electrónico}

Entendemos como pago electrónico aquellas operaciones de pago que se realizan por medios electrónicos. La comisión de las comunidades europeas, por su parte, definen el pago electrónico como cualquier operación de pago efectuada con una tarjeta de pistas magnéticas o con un microprocesador incorporado, en un equipo Terminal de pago electrónico TPE o Terminal de punto de venta (TPV) expresa a las tarjetas electrónicas.

Indicando por "instrumento electrónico de pago" un instrumento que permita a su titular efectuar transacciones, acceder a los fondos de su cuenta en una identidad, autorizando el pago a un beneficiario, quedando incluidas en particular, las tarjetas de pago ( crédito, débito, etc.) y los servicios de tele banca y de banca a domicilio.

\section{Ambigüedades en la Legislación Específica}

En el Código de Comercio en México en el Artículo 80. Los convenios y contratos mercantiles que se celebren por correspondencia, telégrafo, o mediante el uso de medios electrónicos, ópticos o de cualquier otra tecnología, quedarán perfeccionados desde que se reciba la aceptación de la propuesta o las condiciones con que ésta fuere modificada.

No obstante, este Código de Comercio no es claro en cuanto a la legislación específica sobre el pago, por medio de las tarjetas y su análisis jurídico se tiene que hacer sobre el principio de la autonomía de la voluntad de los contratantes y sobre la teoría en general de las obligaciones y los contratos.

La legislación por medio de este artículo, describe entonces que es necesario que exista una propuesta de convenios o contratos mercantiles enviados por medios electrónicos que permita perfeccionar el simple acto mercantil.

Por otro lado, el artículo 89 del mismo Código refuerza lo anterior, permitiendo el uso de medios electrónicos, ópticos o de cualquier otra tecnología en los actos de comercio e indica a su vez una serie de definiciones entre las cuales se consideran las siguientes: destinatario, emisor, firma electrónica, firma electrónica avanzada o fiable, mensaje de datos y sistema de información por referir las más relacionadas en este tema.

De igual forma el artículo 89 bis, protege en cierto modo a los mensajes de datos el cual indica: no se negaran efectos jurídicos, validez o fuerza obligatoria a cualquier tipo de información por la sola razón de que este contenida en un mensaje de datos. (Adicionado mediante decreto publicado en el diario oficial de la federación el 29 de agosto de 2003. n. de e. iij: entra en vigor a partir del 27 de noviembre de 2011 , de conformidad con el artículo primero transitorio del decreto.)

Ahora bien, de acuerdo con lo anterior, que pasaría en una relación de compra venta donde el Emisor es la persona que realiza la compra por internet y envía una serie de datos, tanto personales como de su tarjeta de crédito o débito que es lo que se llama Mensaje de Datos y la empresa que vende se convierte en el Destinatario. Y si se negara el cargo y se involucrarán a las autoridades ¿Cómo se basaría la relación del acto mercantil, el consentimiento, el lugar, el envío, los acuses de recibo y recepción de la información? 
Es decir, que el requisito de muchos sitios de internet de registro y generación de claves o contraseñas, además de brindar una base de datos puede servir como una futura protección en la compra venta por medio de internet ya que serviría como prueba de haber entrado al sistema y realización de la compra (Villegas, s.f).

Lo anterior, solo regula una relación continua entre personas a través de un sistema electrónico donde al existir un monitoreo constante se pueda dar oportunidad y los medios para decir si cierta información, fue o no producida por el consumidor. Los artículos 90 y 91 bis complementan la definición del momento de la recepción de la información tanto en los casos en donde se cuente con un sistema de divulgación como cuando no lo haya.

\section{METODOLOGÍA}

Esta investigación se propone como un estudio no experimental o diseño ex post facto. Se trata de un tipo de investigación en donde no se modifica el fenómeno o situación objeto de análisis, pues la relación entre la variable independiente y la variable dependiente, ya se ha producido con anterioridad, y el investigador sólo puede registrar sus medidas y estudiar esa relación.

El estudio permitió entender al comercio electrónico como una de las principales herramientas de apoyo para el empresario en sus formas de incrementar sus posibilidades de venta y expansión, así como también la optimización de sus recursos con un soporte en las innovaciones científicas y tecnológicas y en particular con el uso del internet en un mercado global.

Población y Muestra

La población objeto de estudio está constituida por una muestra aleatoria de empresarios MIPYMES tanto de tangibles como intangibles de la ciudad de Puebla. La muestra se calculó en un periodo completo de 2012-2013, de acuerdo con las bases de datos de la Secretaria de Economía e INEGI.

Conclusiones a Manera de Propuesta

Estas se presentarán para poder medir la situación actual de los empresarios con respecto al uso del internet para la comercialización de sus productos y para ello, se realizó el siguiente procedimiento:

\section{- DIAGNOSTICO DE LA SITUACIÓN ACTUAL DE LOS EMPRESARIOS}

El diagnóstico se realizó en tres etapas, cada una de éstas pretende identificar el nivel de uso y dominio del comercio electrónico.

\section{Instrumentos}

En la evaluación diagnóstica de la situación actual de las empresas unidades de análisis, se utilizaron los procedimientos de selección, pedido, envío y pago, se realiza totalmente en línea, a través de la misma red mundial, lo cual abarata considerablemente los costos para el fabricante o distribuidor. 
Este tipo de Comercio Electrónico se consideró con empresas de mercancías denominadas "tangibles e intangibles", cuyo ciclo total de venta, pago y entrega puede realizarse por Internet y de manera física, donde para evaluar el comportamiento de las misma se utilizaron encuestas cerradas con indicadores definidos, las habilidades o procedimientos se midieron en el momento de la acción, es decir ejecutando la acción que conlleva cada procedimiento, y además se evaluó la actividad en su conjunto.

La evaluación diagnóstica obligó a utilizar diversos instrumentos e implicar a diferentes agentes. Este trabajo, evaluó a cada empresa por medio de un conjunto de instrumentos que permiten conformar un análisis de cada empresa.

Análisis de los Datos

Los análisis descriptivos que se presentan son para identificar las características de las empresas y de los procedimientos que se realizan en caso de emplear el internet para la comercialización de sus productos.

Y los niveles comparativos son para identificar el uso del comercio electrónico en el nivel de diagnóstico y la evaluación final con el análisis de los datos de cada empresa en relación con las estadísticas que se muestran desfavorables con respecto a los clientes que no consideran el Internet como opción de compra.

\section{RESULTADOS}

Internet en México lo utilizan los jóvenes de entre 15 y 24 años, en su mayoría, de los cuáles el $4.1 \%$ son profesionales, es decir, los que tendrían capacidad de compra y de acuerdo con las estadísticas que se mencionaron, y existiendo en México un mercado menor a los 1.5 millones de los casi 100 millones de mexicanos que conforman el país, nos indica que el futuro del Comercio Electrónico es poco alentador.

Los porcentajes no son en realidad el problema, sino la falta de hábitos para comprar por medios electrónicos, ya que los mexicanos no están muy familiarizados con esta tecnología. Además, existe alto temor por la compra, el miedo a que no llegue el producto al domicilio señalado o que el número de tarjeta de crédito sea interceptado por otra persona, lo anterior está aún latente con fuerza.

El Comercio electrónico es por tanto, a pesar de que las estadísticas se muestran desfavorables, al mismo tiempo nos indican que en un futuro este tipo de comercio tendrá una gran presencia en nuestro país, ya que los que en estos momentos utilizan el Internet, aunque no tengan la posibilidad de compra, (personas entre los 15 y 24 años) se convertirán en los futuros clientes (Flores, 2010).

Se espera que la cultura para comprar por medio electrónico sea más favorable en aceptación, ya que las generaciones de jóvenes crecerán dentro de otro esquema diferente de comercializar y estarán más habituados a este Comercio.

En lo que se refiere a las empresas, el panorama es mucho más alentador que por parte del cliente de forma individual, pues las empresas consideran que el E-Commerce les permitiría reducir sus inventarios, facilitar las relaciones con los clientes para llegar a un mercado más amplio, etc. 
Aunque el Comercio Electrónico no crecerá a un ritmo tan rápido como otros países europeos o como el país vecino de Norte, eso no indica que vaya a desaparecer o fracasar en un futuro; pues se deberá tener cuidado en los puntos básicos informativos que deberá tener el sitio web que pretenda dedicarse a este tipo de comercio.

Pues, las nuevas generaciones (que ahora son las que utilizan el Internet), harán que el Comercio Electrónico deje de ser una novedad, para convertirse en una manera habitual y tradicional de hacer negocios y acrecentar no solo las transacciones comerciales sino también el crecimiento y posicionamiento de las empresas a corto plazo. De igual forma el Comercio Electrónico en México aún tiene muchos campos para explorar y, por lo tanto, permitirá que sobreviva y que alcance un mayor porcentaje de ventas en el tiempo de vida de las empresas mexicanas.

Otro aspecto a tratar, es el área de oportunidad que estos datos representan para muchos negocios de diversos sectores. Las instituciones de crédito hacen importantes esfuerzos para "blindar" al comprador y fomentar el uso de las tarjetas de crédito y débito en línea, lo cual podría facilitar enormemente las transacciones de esa naturaleza.

¿No sería magnífico poder comprar desde la despensa hasta productos no básicos o secundarios con sólo unos clics?, ése es, quizá, uno de los sectores que podría generar un mayor flujo de dinero al tratarse de bienes de consumo regular que representan un gasto relativamente fijo de los consumidores.

\section{CONCLUSIONES}

En la actualidad, el comercio electrónico es una de las principales herramientas para lograr que las empresas incrementen sus posibilidades de venta y expansión, así como también la optimización de sus recursos debido a que cada vez más son los clientes que recurren a la adquisición de productos tangibles o intangibles por internet y esto se ha debido a que se han ampliado las medidas de seguridad, los procesos de seguimiento de órdenes y formas de pago.

Las empresas se encuentran de manera directa o indirecta en una posición de competitividad global y bajo las expectativas de los compradores, y es por eso que en la actualidad estas, deben modificar sus formas de actuar y de organizarse para lograr un cambio general en su cultura organizacional sobre todo en su forma de hacer negocios y romper sus formas arcaicas y aproximarse más sin barreras físicas para estar en contacto con el mundo exterior.

En el E-commerce es necesario recurrir a la Tecnología para generar el Cambio, ya que este establece el vehículo entre los empresarios y sus clientes. Sí consideramos que el Comercio Electrónico permite empresarios más eficientes y flexibles en sus operaciones y, de esa forma se establece una mejor respuesta a las necesidades de los clientes a posicionar sus productos en un mercado global (Turban y King, 2012).

Es conveniente que, los empresarios cambien su concepción con respecto a la forma de incursionar a nuevos mercados y responder de mejor manera a las expectativas de sus clientes, a través del comercio electrónico pero no solo como un contorno complementario, sino como un medio que recurre a la tecnología para el cambio, que genere una relación costo-beneficio. 
Además es importante, tener presente que según Fernández (2000) el comercio electrónico es "cualquier forma de transacción de negocios en la cual las partes interactúan electrónicamente en lugar de intercambios materiales o contacto físico directo", por lo cual se disminuyen los costos de operación para el empresario o del distribuidor o comercializador.

En sus principios, los iniciadores fueron, los creadores independientes de software que comenzaron a ofrecer sus programas como "Shareware", con opción de "bajar" primero una versión inicial de prueba y quien decide adquirirlo se comunica con el propietario y éste le envía una clave para activar todo el programa, o se lo envía por e-mail previo pago por medio electrónico.

Posteriormente, siguieron las casas editoras y actualmente todos los países han favorecido las cifras estadísticas que denotan los mayores beneficios, como es el caso de las reservaciones turísticas y reservaciones de pasajes de avión y otros transportes por Internet, en la cual ya están incursionando algunas empresas de productos intangibles.

Por consiguiente, el comercio electrónico permite:

- Disminución e incluso eliminación del costo de impresión de catálogos de venta.

- Disminución de los costos en las actualizaciones de catálogos de venta

- Incursionar en nuevos mercados potenciales

- Elimina la exigencia de visitas y coordinaciones para escoger mercancías.

- Mejora la comunicación con los clientes.

- En la promoción productos intangibles como es el caso de las reservaciones turísticas y de transportación, fotos, videos, música, información y software, no se requiere infraestructura logística.

- Se puede recurrir a la promoción de manera más sofisticada como es usando técnicas mercadológicas a mucho menor costo que los anuncios hechos en empresas de medios de difusión.

- $\quad$ Aumento en el acceso cada día de más nuevos cibernautas

Por tanto, se incrementan las posibilidades de venta directa al consumidor sin necesidad de pasar por intermediarios no solo en países de alto desarrollo, sino; que actualmente se ha logrado en países de América Latina donde los clientes se han permitido realizar compras desde su caso o la oficina.

De forma más reciente se realizan ahora hasta subastas en línea, donde cualquier particular sin necesidad de una casa de venta o sitios comerciales, donde se pueden colocar a la venta productos en un sitio web como es mercado libre por citar a uno de los tantos que existen.

Existen algunas dificultades en los países latinoamericanos que hacen que se debiliten ante el e-commerce como es la infraestructura en sus comunicaciones, falta de acceso a computadoras por parte de sus habitantes, índices bajos de conectividad y poca cultura de compra sin visita física al establecimiento. 
Sin embargo, aún con todos estos inconvenientes las empresas se están incursionándo cada vez más al comercio electrónico, sin importar su ubicación geográfica, las características de su región o el sector al que pertenecen, y si su enfrentamiento es con grandes empresarios o a empresas que ya tienen nichos de mercado.

Por tanto, las herramientas tecnológicas permiten lograr un salto en la competitividad de la economía de las empresas y alcanzan así mejores niveles de vida sin moverse de la oficina o la casa y con menos costo que el uso del teléfono o fax. De hecho, el reto de las empresas es que estas puedan operarse con menores costos para que los clientes accedan a sus productos e incursionar en nuevas formas de negociación y liderazgo ante la competencia para lograr mejoras también en la productividad.

Sin embargo, aún hace falta adecuar el marco jurídico y sumarse a las voluntades de los empresarios, sus organizaciones, el público en general y las universidades para convocar a la acción de todos en función de cerrar la brecha digital que se ha generado entre la economía y el uso de las nuevas tecnologías de la información.

\section{REFERENCIAS BIBLIOGRÁFÍCAS}

Asociación Mexicana de Internet (AMIPCI) (2012). "Hábitos de los usuarios de internet en México". Recuperado 10/12/2013 de: http://www.amipci.org.mx/estudios/ habitos_de_internet/2012HabitosUsuariosInternetMx.pdf

Cámaras. (2005). “Tecnologías de la información. Aspectos jurídicos”. Recuperado 25/11/2013 de: http://www.camaras.org/publicado/estudios/pdf/otras_pub/manual_ tic/manual_tic_2004.pdf

Carrol, J. y Broadhead, R. (2000). "Selling On-Line, Ed. MacMillan, Toronto, Recuperado 30/08/2013 de: tpn.geis.com/tpn/resource_center/casestud.html.

El Economista. (2012). "Comercio electrónico en México crecerá 46\% en el 2012". Recuperado 03/12/2013 de: http:/eleconomista.com.mx/tecnociencia/2012/11/06/ comercio-electronico-mexico-crecera-46-2012

Fernández, J. (2000). Coordinador de la Comisión Nacional de CE de Cuba. Conferencia sobre el Comercio Electrónico en Cuba, Taller Nacional de CE, La Habana.

Financial Times on-line. (2001). News Analysis, B2B Issues, "Aiming for global leadership in B2B marketplace”. Recuperado 10/12/2012 de: www.ft.com

Flores, S. (2010). "Implementación del comercio electrónico y el teletrabajo como motor impulsor del crecimiento en las pymes de México. Universidad Veracruzana. Recuperado 25/08/2013 de: http://cdigital.uv.mx/bitstream/123456789/28507/1/ Flores\%20Flores.pdf

ILifebelt Times On-line. (2013). Usuarios y uso de Internet en México 2013. Recuperado 15/08/2013 de: http://ilifebelt.com/usuarios-y-uso-de-internet-enmexico.

Kenneth, C. y Carol, G. (2012). E-Commerce. ed.8 ${ }^{a}$. Ed. Business. Technology. Society 
Lagarde, H. (s.f). México: el comercio electrónico un reto. Razón y palabra. Recuperado 12/01/2014 de: http://www.razonypalabra.org.mx/anteriores/n20/20_ hlagarde.html

Lazcano, L. (2006). El comercio electrónico en México: una realidad o una proyección a futuro. Universidad Autónoma Metropolitana. Recuperado 25/1 1/2013 de: http://148.206.53.84/tesiuami/UAMI13654.pdf

Liberos, E. y García, R. (2012). El libro del Comercio Electrónico. ed. 2a . Ed. Libros Profesionales de Empresas (ESIC Marketing \& Business School a través del ICEMD)

López, M. y Pérez, S. (s.f). "Comercio electrónico: una nueva cultura de hacer negocios”. Gestión y estrategia. Recuperado 25/01/2014 de: http://administracion. azc.uam.mx/descargas/revistagye/rv20/rev20art07.pdf

Moreno, M. (s.f). El futuro del comercio electrónico en México. Recuperado 10/01/2014 de: http://www.marketingycomercio.com/n25jun01/14jun01b.htm

Redherring Communications. (2001). Recuperado 12/12/2013 de: www.redherring. com - Industry Profiles

Turban, E. y King, D. (2012). Electronic Commerce. Ed. Pearson. Global. Ed. $7^{\mathrm{a}}$.

Villegas. (s.f). "Marco jurídico del comercio electrónico en México". Recuperado 15/01/2014 de: http://www.itescam.edu.mx/principal/sylabus/fpdb/recursos/r54194. PDF 